Les ANNALES Les Annales de droit

DE DROIT

13 | 2019

Varia

\title{
Pour une responsabilité civile européenne élargie des agences de notation de crédit
}

For enlarging a European, civil responsibility of credit rating agencies

Farouk Bellil

\section{(2) OpenEdition}

Journals

Édition électronique

URL : http://journals.openedition.org/add/1422

DOI : 10.4000/add. 1422

ISSN : 2606-1988

Éditeur

Presses universitaires de Rouen et du Havre

Édition imprimée

Date de publication : 1 mai 2019

Pagination : 29-55

ISBN : 979-10-240-1293-3

ISSN : 1955-0855

Référence électronique

Farouk Bellil, « Pour une responsabilité civile européenne élargie des agences de notation de crédit », Les Annales de droit [En ligne], 13 | 2019, mis en ligne le 09 décembre 2019, consulté le 25 janvier 2021. URL : http://journals.openedition.org/add/1422 ; DOI : https://doi.org/10.4000/add. 1422 


\title{
Pour une responsabilité civile européenne élargie des agences de notation de crédit
}

\author{
Farouk BeLLIL
}

Les toutes premières sanctions prononcées par l'autorité européenne des marchés financiers à l'encontre des agences de notation de crédit, ainsi que les récentes mises en cause de leur responsabilité civile aux États-Unis et en Australie invitent à s'interroger sur l'efficacité de la réglementation européenne en la matière. L'instauration d'un régime de responsabilité limité des agences de notation par le règlement 462/2013 peut-il permettre d'aboutir à des décisions semblables? La question est essentielle tant au regard du rôle fondamental des agences dans l'évaluation du risque de crédit, que de leur influence sur la confiance et les décisions prises par les investisseurs sur les marchés. Envisager la mise en place d'une obligation de diligence des agences de notation pourrait permettre une mise en œuvre effective de leur responsabilité.

Simples boucs émissaires ou véritables participants au déclenchement de la crise financière de 2007 , les agences de notation font désormais l'objet d'une attention toute particulière de la part des politiques nationales et internationales. Apparues à la fin du $\mathrm{XIX}^{\mathrm{e}}$ siècle, les agences de notation de crédit sont des sociétés commerciales dont l'activité consiste à attribuer une note à toutes entités pouvant émettre des titres obligataires, qu'il s'agisse d'une société ou d'un État ${ }^{1}$. Cette notation a pour objet de renseigner les investisseurs de manière synthétique quant à la qualité du crédit de l'entité à laquelle ils entendent prêter. Elle permet ainsi d'évaluer le risque de non-paiement d'un émetteur ou d'un instrument financier particulier en se fondant sur des méthodes de calculs standardisées aboutissant à l'attribution d'une note. Un investisseur n'a donc plus à évaluer lui-même le risque que présente un instrument financier, mais simplement à déléguer cette tâche à une agence de notation.

1. Plus généralement sur les agences de notation: Kim Oosterlinck, «Rôle et nécessité des agences de notation: une perspective historique» dans Bruno Colmant (dir.), Les agences de notation financière entre marchés et États, Bruxelles, Larcier, 2013, p. 17 . 
Les agences de notation jouent aujourd'hui un rôle central dans l'économie mondiale, et cela au moins pour deux raisons principales. D'une part, les notations constituent un rouage essentiel de la régulation financière mondiale. Elles sont en effet utilisées à des fins réglementaires, aussi bien aux États-Unis qu'en Europe ${ }^{2}$. À titre d'exemple, l'article 18.2 de la directive $2006 / 73^{3}$ définit la haute qualité d'un instrument du marché monétaire comme celui qui a reçu de toutes les agences de notation la meilleure note possible. Ainsi, les fonds du marché monétaire qualifié ne sont autorisés à investir que dans les instruments ayant reçu les meilleures notations ${ }^{4}$. D'autre part, les notations dictent véritablement le comportement des investisseurs. Les agences de notation sont devenues des acteurs incontournables des marchés financiers, une note défavorable pouvant conduire un emprunteur à de graves difficultés.

Il y a encore une vingtaine d'années, les agences de notations étaient comparées à une super puissance pouvant anéantir un État en dégradant sa note ${ }^{5}$, il semble que la crise ait conduit à atténuer cette affirmation. En effet, suite à l'annonce du Brexit certains observateurs ${ }^{6}$ se demandaient si les agences de notation avaient encore une influence. La question était légitime puisqu'à l'annonce de la sortie du Royaume-Uni de l'Union européenne, les trois principales agences de notations n'ont pas hésité à dégrader la note de cet État faisant passer celle-ci du fameux «triple A » $\mathrm{au}$ « $\mathrm{AA}$ ». Logiquement, une telle dégradation aurait dû entraîner une augmentation des coûts d'emprunt pour le Royaume-Uni, ce qui ne fut pas le cas. Une situation similaire s'était déjà produite avec la dégradation de la note des États-Unis en 2011. Il apparaît ainsi que les marchés tendent davantage à relativiser les changements de notation en ce qui concerne les dettes souveraines. La question demeure cependant tout autre en ce qui concerne les obligations émises dans le secteur privé.

2. Voir Mikael Petitjean, "De l'(in)utilité des agences de notation», Regards économique, $\mathrm{n}^{\circ} 98,2012$.

3. Dir. 2006/73/CE, 10 août 2006, portant mesures d'exécution de la PE et Cons. UE, dir. 2004/39/CE, en ce qui concerne les exigences organisationnelles et les conditions d'exercice applicables aux entreprises d'investissement et la définition de certains termes aux fins de ladite directive (texte présentant de l'intérêt pour l'EEE), JOUE $\mathrm{n}^{\mathrm{o}} \mathrm{L} 241,2$ sept. 2006, p. 26.

4. Sur l'utilisation des notations à des fins réglementaires, voir Edith Weemaels, "Les agences de notation en Europe: contrôle, responsabilité et légitimité», dans Brunot Colmant (dir.), op. cit., p. 63, spéc. p. 104 et suiv.

5. Thomas L. Friedman, «Foreign affairs, Don't mess with Moody's ", New York Times, 22 février 1995.

6. Elaine Moore, «Do Sovereign credit ratings still matter?», Financial Times, 14 juillet 2016. 
En effet, pour une société cherchant à se financer, la notation demeure essentielle et va avoir un effet direct sur son financement en influant sur les taux d'intérêt. La notation s'avère également déterminante pour les investisseurs : ils vont bien souvent fonder leurs décisions d'investir sur les seules notations, externalisant ainsi la responsabilité de leur jugement ${ }^{7}$. Une notation qui ne refléterait pas la réalité économique d'un produit financier ou d'une société pourrait avoir de très lourdes conséquences financières tant pour les investisseurs que pour ladite société. Cette question ne concerne pas directement les agences de notation extra-financières, compte tenu des critères d'évaluation qu'elles utilisent et de leur absence d'implication dans la crise financière ${ }^{8}$.

Après à la crise financière de 2007 , la pertinence des notations a commencé à être remise en cause. Il s'est avéré que l'activité des agences était trop souvent affectée par des conflits d'intérêts, la notation émise étant payée par l'émetteur et non par l'investisseur ${ }^{9}$. De plus, certaines agences avaient attribué de très bonnes notes à des produits financiers dits toxiques, précipitant ainsi la chute de nombreux investisseurs. La réaction des États ne s'est pas fait attendre, notamment aux États-Unis, marché historique des agences, engageant un véritable "bras de fer ${ }^{10}$ " avec ces dernières. Outre les réactions politiques parfois virulentes, un cadre législatif restreignant l'emprise des agences de part et d'autre de l'Atlantique a vu le jour. Le Dodd-Franck Act adopté en juillet 2010 par le Congrès américain, ainsi que le règlement 1060/2009 ${ }^{11}$ poursuivent des objectifs similaires: limiter la dépendance réglementaire aux notations, stimuler la concurrence entre les agences, accroitre leur transparence, mais surtout réglementer leurs activités en les supervisant et en sanctionnant les éventuels manquements. Ces différentes dispositions

7. Jean-Marc Gollier, "Le courage de la vérité dans un monde mimétique», dans Brunot Colmant (dir.), op. cit., p. 143.

8. Thierry Garnier, "L'absence de responsabilité des agences de notation extrafinancière?», Bull. Joly Bourse 2014, $\mathrm{n}^{\circ} 9$.

9. Hubert de Vauplane, «Responsabilité des agences de notation: état des lieux en Europe et aux États-Unis", Revue Banque, $\mathrm{n}^{\circ} 746$, mars 2012, p. 78 . Hubert de Vauplane, "Pour une responsabilité civile entière des agences de notation", Mélanges AEDBF, $\mathrm{n}^{\circ}$ 5, 2008, p. 449.

10. Gregory Lewkowicz, «Les agences de notation financière contre les États : une lutte globale pour le droit à l'issue incertaine », dans Brunot Colmant (dir.), op. cit, p. 185, spéc. p. 186.

11. Comm. CE, règl. (CE) $n^{0} 1060 / 2009,16$ sept. 2009 sur les agences de notation, JOUE $\mathrm{n}^{\mathrm{o}} \mathrm{L}$ 302, 17 nov. 2009, p. 1. Ivan Tcotourian, «Le règlement (CE) $\mathrm{n}^{\mathrm{o}}$ 1060/2009 concernant les agences de notation de crédit: vrai réforme ou faux-semblant? ", Bull. Joly Bourse, ${ }^{\text {er }}$ septembre 2010, $\mathrm{n}^{\text {o }}$ 5, p. 430. 
aboutissent cependant à une situation assez paradoxale : en renforçant le contrôle des agences, l'Union et les États-Unis ont indirectement institutionnalisé et consolidé leur rôle ${ }^{12}$.

Eu égard à l'importance acquise par les agences dans le fonctionnement des marchés, seule une responsabilité effective de ces dernières est de nature à leur conférer une véritable légitimité. L'enjeu est essentiel: un investisseur qui aurait subi des pertes en ayant fondé sa décision d'investir sur une notation erronée pourrait-il obtenir réparation? De même, un émetteur qui se serait vu attribuer une notation non méritée pourrait-il également obtenir réparation? Comment déterminer ce qu'est une notation fautive? Sur quels critères objectifs pourrait-elle être jugée et permettre d'établir un lien de causalité avec le préjudice allégué? Autrement dit, quel régime de responsabilité convient-il d'adopter pour les agences de notation de crédit? Sur ces questions, le droit américain n'est pas réellement apparu efficace ${ }^{13}$. Le Dodd-Frank Act de 2010 crée un droit d'action en responsabilité contre une agence qui n'aurait pas mis en œuvre, en connaissance de cause ou par négligence, des moyens raisonnables d'information sur les produits financiers soumis à son évaluation. Dans le cadre d'une action en responsabilité, les agences se voient seulement imposer de divulguer la méthodologie et les éléments factuels sur lesquels elles se sont appuyées pour rendre leurs notations. De plus, le plaignant ne peut obtenir réparation qu'à la condition de démontrer que l'agence a émis la notation en sachant qu'elle était fausse ou avec une insouciance téméraire par rapport à la réalité ${ }^{14}$. Surtout, cette responsabilité ne concerne que les notations figurant dans les déclarations d'inscription ou les prospectus des émetteurs. Or, bien qu'il s'agisse d'une obligation légale, les agences refusent désormais, suite à l'adoption de cette disposition, que les émetteurs fassent mention des notes attribuées à tout produit financier structuré dans les prospectus, ce qui de fait, les met à l'abri de toute action en responsabilité ${ }^{15}$. Ce mouvement a contraint la Securities and Exchange Commission (SEC) à suspendre les effets du $₫ 933(\mathrm{~m})(1)$ qui impose aux émetteurs de joindre

12. Gregory Lewkowicz, op. cit.

13. Carrie Guo, "Credit Rating Agency Reform: A Review of Dodd-Frank Section 933(B)'s Effect (Or Lack Thereof) Since Enactment», Columbia Business Law Review, $\mathrm{n}^{\circ}$ 184, 2016.

14. Alexandre Hublet, «Le paradigme d'un paradoxe : la délégation de compétences aux agences de notation par les États en l'absence d'un contrôle réglementaire», dans Brunot Colmant (dir.), op. cit., p. 39, spéc. p. 51-52.

15. Edith Weemaels, «Les agences de notation en Europe : contrôle, responsabilité et légitimité », dans Brunot Colmant (dir.), op. cit., p. 63, spéc. p. 99 et 100. 
une notation dans les dossiers qui leur étaient transmis avant qu'elle puisse autoriser l'émission de titres adossés à des actifs ${ }^{16}$, remettant ainsi en cause l'efficacité des dispositions nouvellement adoptées. Ces questions ne concernent pas directement les agences de notation extrafinancières compte tenu de la nature de ces dernières, qui prennent en compte des critères d'évaluation très variés, et de leur absence d'implication dans la crise financière de $2007^{17}$.

$\mathrm{Au}$ regard de la difficulté qu'il peut y avoir à établir la responsabilité civile des agences, certains suggèrent de seulement publier les infractions des agences dans la presse économique plutôt que de mettre en place un régime de responsabilité spécifique ${ }^{18}$. Cette idée ne semble pas convaincante, essentiellement pour deux raisons. D'une part, la réglementation européenne instaure déjà une responsabilité administrative des agences par le biais de sanctions prononcées et publiées par l'autorité européenne des marchés financiers (AEMF) en cas d'infractions aux dispositions du règlement 1060/2009. D'autre part, la responsabilité civile poursuit une double fonction : une fonction réparatrice qui oblige à dédommager le préjudice causé à autrui, et une fonction normative contribuant à la prévention des comportements qui enfreindraient la loi ${ }^{19}$. C'est pourquoi l'instauration d'une responsabilité civile des agences de notation est essentielle. Sans l'instauration d'un tel régime, les victimes d'une notation erronée ne pourraient pas obtenir réparation, et les agences ne seraient pas incitées à faire preuve davantage de rigueur lors du processus de notation. Face à ces enjeux, le législateur européen a tenté de mettre en place un régime de responsabilité civile des agences de notation. Si une action en réparation à l'encontre de ces dernières devient possible, elle demeure en pratique limitée et difficile à mettre en œuvre. Les règles européennes en la matière s'avèrent ainsi peu efficaces (1). Sans remettre en cause la nature même de l'activité de notation (prévoir un événement futur), imposer une obligation de diligence aux agences de notation

16. Gregory Lewkowicz, op. cit., p. 185, spec. p. 211. Benjamin H. Brownlow, «Rating Agency Reform: Preserving The Registered Market For Asset-Backed Securities ", North Carolina Banking Institute, vol. $15, \mathrm{n}^{\circ}$ 1, 2011, p. 111 et suiv.

17. Thierry Garnier, "L'absence de responsabilité des agences de notation extrafinancière?", Bull. Joly Bourse 2014, $\mathrm{n}^{\circ} 9$.

18. Norbert Gaillard, "Quelle réglementation pour l'industrie de la notation?», SciencesPo, décembre 2012, www.sciencespo.fr/ceri/sites/sciencespo.fr.ceri/files/ notation_ng.pdf.

19. Clothilde Grare, Recherche sur la cohérence de la responsabilité civile délictuelle. L'influence des fondements de la responsabilité sur la réparation, Paris, Dalloz, 2005, $\mathrm{n}^{\mathrm{o}} 390$ et suiv., $\mathrm{n}^{\mathrm{o}} 442$ et suiv. 
peut paraître la solution la mieux à même d'assurer une mise en œuvre effective de leur responsabilité civile (2).

\section{Des règles européennes peu efficaces pour établir une responsabilité civile des agences de notation}

Le règlement $462 / 2013^{20}$ révise le règlement $1060 / 2009$ et introduit un titre III bis relatif à la responsabilité des agences de notation. Son article 35 bis harmonise les règles relatives à la responsabilité civile des agences de notation entre les États membres (1.1). Toutefois, il s'agit d'une responsabilité limitée, pouvant être complétée par «le droit national compétent selon les règles de droit international privé applicables». Le droit européen repose ainsi en partie sur le droit national, afin d'assurer une responsabilité civile effective des agences de notation. Encore faut-il que le droit national compétent puisse clairement être identifié, ce qui pose également des difficultés (1.2).

\subsection{L'article 35 bis du règlement 462/2013: une responsabilité civile limitée}

Le régime de responsabilité mis en place par le règlement 462/2013 présente une utilité certaine au regard de la disparité existante entre les différentes législations nationales avant son adoption (1.1.2). Il s'avère néanmoins incomplet et difficile à mettre en œuvre, ce qui limite grandement son efficacité (1.1.2).

\subsubsection{La nécessaire mise en place d'un régime de responsabilité des agences de notation au niveau européen}

L'un des principaux apports du règlement $462 / 2013$ consiste en la mise en place d'un régime de responsabilité civile des agences de notation de crédit. Auparavant, le droit de l'Union se désintéressait de cette question, abandonnant cette prérogative aux États membres. Le règlement 1060/2009 précisait simplement que «tout recours visant des agences de notation de crédit en relation avec une violation des dispositions du présent règlement devrait être effectué conformément au droit national applicable en matière de responsabilité civile » (considérant 69). Dès lors, les conditions de la responsabilité des agences

20. Comm. UE, règl. (UE) $n^{0} 462 / 2013$ du Parlement européen et du Conseil du 21 mai 2013 modifiant le Comm. CE, règl. (CE) $n^{0}$ 1060/2009 sur les agences de notation de crédit. Texte présentant de l'intérêt pour l'EEE, JOUE ${ }^{\circ} \mathrm{L}_{146} 31$ mai 2013, p. 1. 
relevaient des différents droits nationaux. À la suite de l'entrée en vigueur du règlement 1060/2009, le législateur français s'est empressé d'adopter des règles en la matière ${ }^{21}$. Sans revenir sur la pertinence de ces dispositions, il convient de relever que la situation globale au sein de l'Union n'était pas satisfaisante, la mise en œuvre de la responsabilité des agences pouvant grandement varier d'un État membre à l'autre. Certains États comme la Pologne ou la Suède ne permettaient aucune action en responsabilité civile à l'égard des agences de notation ${ }^{22}$. En instaurant un régime de responsabilité des agences au niveau européen, le législateur a ainsi souhaité éviter les différences de traitement entre les investisseurs susceptibles de favoriser le forum shopping : les agences peuvent choisir la loi applicable au contrat ou s'installer dans les États membres où la législation en matière de responsabilité leur est plus favorable.

La Commission des affaires juridiques du Parlement européen ${ }^{23}$ souhaite également faciliter la réparation du préjudice subi par un investisseur qui se serait fondé sur une notation émise en violation des dispositions du règlement 1060/2009 et qui n'aurait pas de relation contractuelle directe avec l'agence de notation. Ce dernier pourrait se voir privé de réparation malgré l'existence d'une faute résultant d'une violation du règlement 1060/2009. L'article 35 bis du règlement 462/2013 répond à ces attentes en instaurant un régime commun de droit matériel de la responsabilité civile des agences. Dans les contrats les liant à leurs clients, les agences déterminent le droit applicable en cas de litige, en s'assurant que ce dernier leur soit plus favorable. La mise en œuvre de la responsabilité civile contractuelle des agences s'avère ainsi limitée. Afin de contourner ces difficultés, le règlement 462/2013 prévoient la possibilité pour l'émetteur, qui aurait conclu un contrat avec une agence, de fonder son action en réparation non pas sur une disposition contractuelle, mais sur un manquement aux dispositions du règlement 1060/2009 (considérant 32).

Le législateur a tenté d'atteindre un juste équilibre entre les différents intérêts en présence, en s'assurant que la responsabilité des agences ne peut pas être invoquée de manière «illimitée» (considérant 33). Toutefois, en procédant ainsi le législateur européen a mis en place un régime de responsabilité incomplet.

21. C. monét. fin., art. L. 544-5 et L. 544-6.

22. Avis de la Commission des affaires juridiques à l'intention de la Commission des affaires économiques et monétaires sur les agences de notation de crédit: perspectives d'avenir, $1^{\text {er }}$ mars 2011, 2010/2302(INI).

23. Ibid. 


\subsubsection{Un régime de responsabilité incomplet}

L'action en réparation sur le fondement de l'article 35 bis ne peut-être intentée que :

lorsqu'une agence de notation de crédit, de manière intentionnelle ou par négligence grave a commis l'une des infractions énumérées à l'annexe III et que cette infraction a eu une incidence sur une notation de crédit, un investisseur ou un émetteur peuvent demander réparation à cette agence de notation de crédit pour le préjudice qu' ils ont subi du fait de cette infraction.

Dès lors, le régime de responsabilité civile mis en place par le règlement est incomplet puisqu'il vise seulement à réparer les préjudices résultants d'une infraction à l'annexe III du règlement. De plus, les conditions de la mise en œuvre de cette disposition sont multiples et difficiles à réunir.

L'agence doit tout d'abord avoir commis une infraction énumérée à l'annexe III du règlement, que ce soit de manière intentionnelle ou par négligence grave. L'annexe contient une liste limitative de 73 infractions, comme la violation des règles liées aux conflits d'intérêts ou aux exigences organisationnelles. Indépendamment d'une action en responsabilité civile sur le fondement de l'article 35 bis, les infractions listées peuvent être sanctionnées par l'AEMF aux moyens de lourdes amendes, d'astreintes (art. 36 bis et ter) et en ce qui concerne les infractions les plus graves, par un retrait de l'enregistrement d'une agence (art. 24). À titre d'exemple, en juillet 2016, l'AEMF a infligé une amende de 1,38 million d'euros à Fitch en raison d'une série d'infractions par négligence au règlement 1060/2009 commise entre juin et décembre $2012^{24}$. Les sanctions prononcées par l'AEMF sont essentielles afin d'assurer le respect de la réglementation prudentielle et en ce qu'elles permettent aux investisseurs et aux émetteurs de fonder leurs actions en réparation. En effet, l'article 35 bis $2 \mathrm{du}$ règlement dispose qu'il «incombe à l'investisseur ou à l'émetteur d'apporter des informations précises et circonstanciées indiquant que l'agence de notation de crédit a commis une infraction au présent règlement, et que cette infraction a eu une incidence sur la notation de crédit émise». Dès lors, la charge de la preuve de l'existence d'une infraction au règlement qui peut fonder l'action en responsabilité de l'émetteur ou de l'investisseur pèse sur ces derniers. Or, de telles informations semblent difficilement accessibles aux investisseurs ou aux émetteurs étant donné qu'elles demeurent « purement dans la sphère de

24. ESMA, communiqué du 21 juillet 2016, ESMA/2016/115, https://www.esma.europa.eu/sites/default/files/library/2016-1157_press_release__esma_fines_fitch.pdf. 
l'agence de notation de crédit» (art. 35 bis 2). Face à cette difficulté, le règlement précise simplement qu'il appartiendra «à la juridiction nationale compétente d'apprécier le caractère précis et circonstancié des informations". Ainsi, en dehors de l'hypothèse où une agence serait condamnée par l'AEMF, il semble difficile, voire impossible pour un investisseur ou un émetteur d'établir l'existence d'une infraction au règlement 1060/2009 pouvant fonder son action en responsabilité à l'égard d'une agence de notation. Or, le nombre de condamnations prononcées par l'AEMF risque de se révéler faible au regard de ses effectifs ( 36 employés) et des différentes tâches qui lui sont dévolues ${ }^{25}$.

Même en cas de condamnation d'une agence par l'AEMF, l'investisseur ou l'émetteur victime n'est pas certain de pouvoir engager une action en responsabilité. En effet, de manière classique, le règlement exige de démontrer que l'infraction ait eu « une incidence» sur la notation. Or, la démonstration du lien causal entre l'existence d'une infraction au règlement et un préjudice n'est pas toujours évidente. Un manquement à l'une des obligations de l'annexe III du règlement 1060/2009 n'a pas nécessairement une influence directe sur la notation attribuée. À titre d'exemple, si une agence omet d'opérer une rotation de ses analystes conformément au règlement (art. 7.4), elle commet une infraction qui peut être sanctionnée par l'AEMF, mais rien ne prouve que cette omission a eu une quelconque incidence sur la qualité de la notation ${ }^{26}$.

Il faut en outre que l'émetteur victime d'une notation erronée démontre que l'infraction commise par l'agence qui a affecté son produit n'a pas été causée par des informations trompeuses ou inexactes qu'il aurait lui-même fournies à l'agence (art. 35 bis 1). L'investisseur devra de son côté démontrer «qu'il s'est raisonnablement appuyé sur une notation de crédit, conformément à l'article 5 bis, paragraphe 1, ou autrement en faisant preuve de toute la diligence requise, pour décider d'investir dans l'instrument financier couvert par cette notation de crédit, de le détenir ou de le céder ». Le règlement semble ainsi mettre en place une obligation de prudence à l'égard des investisseurs: ces derniers sont incités à ne pas se fier aveuglément aux notations et à évaluer euxmêmes la qualité du crédit ${ }^{27}$. Cette obligation vaut essentiellement pour un certain nombre d'investisseurs qui ont la capacité de procéder à ce

25. AEMF, 2013, work programme, ESMA/2012/631, 5 avril 2013, p. 9.

26. Anastasia Sotiropoulou, "La responsabilité civile des agences de notation», Bull. Joly Bourse 2013, $\mathrm{n}^{\circ}$ 1, p. 47, spéc. $\$ 18$.

27. Art. 5 bis du $\mathrm{PE}$ et Cons. UE, règl. $\mathrm{n}^{\circ}$ 462/2013: Dépendance excessive des institutions financières à l'égard des notations de crédit. 
type d'expertise, comme les établissements de crédit ou les entreprises d'investissement ${ }^{28}$. Pour les titres à propos desquels la notation attribuée est l'unique source d'information, comme les nouveaux produits par exemple, la condition relative à la diligence de l'investisseur ne devrait pas poser de difficultés. Toutefois, pour les produits dont la consistance est plus aisée à percevoir, le risque existe, même en cas de violation du règlement 1060/2009, que l'action en réparation soit rejetée.

On le voit, les obstacles juridiques à l'établissement de la responsabilité civile des agences de notation sur le fondement du règlement européen sont nombreux. Il semble que le législateur européen ait tenté d'atteindre un équilibre entre l'introduction d'un cadre de responsabilité des agences et le besoin d'éviter l'écueil de rendre le marché européen dissuasif pour les nouveaux entrants ${ }^{29}$. Toutefois, il n'est pas certain que le régime ainsi mis en place parvient à satisfaire cet équilibre. En effet, ce n'est pas la notation trompeuse qui est sanctionnée, mais seulement les infractions à l'annexe III du règlement 1060/2009. Dès lors, le règlement ne pose pas un régime de responsabilité complet et prévoit un renvoi au droit national sur de nombreuses questions. L'article 35 bis 5 indique que «le présent article n'exclut pas d'autres actions en responsabilité civile conformément au droit national ». En dehors des préjudices résultants d'une violation de l'annexe III, seul le droit national sera compétent. Il est regrettable que le règlement, qui entend pourtant procéder à une harmonisation des règles relatives à la responsabilité civile des agences de notation, aboutisse finalement à mettre en place un régime de responsabilité à double vitesse, selon le droit national applicable. Ce dernier est « déterminé selon les règles applicables du droit international privé » (art. 35 bis 4).

28. L'art. 4.1 du PE et Cons. UE, règl. $\mathrm{n}^{\circ} 462 / 2013$ vise «les établissements de crédit, les entreprises d'investissement, les entreprises d'assurance, les entreprises de réassurance, les institutions de retraite professionnelle, les sociétés de gestion, les sociétés d'investissement, les gestionnaires de fonds d'investissement alternatifs et les contreparties centrales ne peuvent utiliser à des fins réglementaires que des notations de crédit émises par des agences de notation de crédit établies dans l'Union et enregistrées conformément au présent règlement».

29. Brigitte Haar, "Civil Liability of Credit Rating Agencies after CRA 3 - Regulatory All-or-Nothing Approaches between Immunity and Over-Deterrence», University of Oslo Faculty of Law Legal Studies Research Paper, $\mathrm{n}^{\mathrm{o}}$ 2013-02, 2013, p. 11 et suiv., http://ssrn.com/abstract=2198293. 


\subsection{L'inadaptation des règles de conflit européennes en matière de responsabilité civile des agences de notation}

La détermination de la loi applicable à l'action en responsabilité à l'égard des agences de notation est essentielle à double titre. D'une part, les termes de "préjudice, intention, négligence grave, raisonnablement appuyée, diligence requise, incidence, raisonnable et proportionnée ", visés à l'article 35 du règlement, sont interprétés et appliqués conformément au droit national applicable (art. 35 bis 4). D'autre part, les questions de responsabilité civile des agences de notation qui ne relèvent pas du règlement 462/2013 sont également régies par ledit droit national. Or, les règles de conflits de lois mises en place par le droit de l'Union s'avèrent mal adaptées à la responsabilité civile des agences de notation, ce qui soulève des difficultés d'interprétation (1.2.1). Une interprétation tenant compte des objectifs fixés par le législateur européen et de différents intérêts en présence doit être dégagée (1.2.2).

\subsubsection{Les hésitations relatives à l'identification de la loi du lieu du dommage}

Les litiges en matière de responsabilité des agences intervenant essentiellement dans un contexte international et le droit de l'Union renvoyant en partie sur cette question au droit national, il est essentiel de déterminer la loi applicable à l'action en responsabilité du souscripteur à l'égard de l'agence. Le règlement 462/2013 indique seulement que «les questions de responsabilité civile qui concernent les agences de notation de crédit, qui ne sont pas couvertes ou définies par le présent règlement, $y$ compris la causalité et la notion de négligence grave, devraient être régies par le droit national applicable déterminé selon les règles pertinentes du droit international privé » (considérant 35). La référence aux « règles pertinentes de droit international privé », doit être comprise comme renvoyant aux dispositions du règlement $864 / 2007^{30}$ (Rome II) qui unifie les règles de conflit de lois en matière d'obligations non contractuelles. Une partie de la doctrine ${ }^{31}$ avait cru, en se fondant sur une interprétation

30. Comm. CE, règl. (CE) $n^{0} 864 / 2007,11$ juill. 2007, sur la loi applicable aux obligations non contractuelles (Rome II), JOUE $n^{\circ}$ L 199, 31 juill. 2007, p. 40.

31. Mathias Audit, «Aspects internationaux de la responsabilité civile des agences de notation», RCDIP, 2011, p. 581, spéc. $\$ 22$ et suiv. 
du considérant 69 du règlement $1060 / 2009^{32}$ et de l'article L. $544-5 \mathrm{du}$ code monétaire et financier français ${ }^{33}$, pouvoir écarter l'application du règlement Rome II dès lors que le siège social de l'agence fautive était situé en France. Cette solution semble contestable dès lors que l'article 27 du règlement Rome II indique que «le présent règlement n'affecte pas l'application des dispositions de droit communautaire qui, dans des matières particulières, règlent les conflits de lois en matière d'obligations non contractuelles». Le règlement 462/2013 clarifie désormais l'interprétation du considérant 69 du règlement 1060/2009, puisqu'il n'instaure aucune règle de conflits de lois en matière de responsabilité délictuelle. Ainsi, ce sont bien les dispositions du règlement Rome II qui doivent s'appliquer. Celui-ci ne comporte aucune règle spécifique concernant la question de la responsabilité civile des agences de notation et plus généralement en matière financière ${ }^{34}$, la règle générale prévue à l'article $4.1 \mathrm{du}$ règlement devrait donc s'appliquer. Cet article dispose que « la loi applicable à une obligation non contractuelle résultant d'un fait dommageable est celle du pays où le dommage survient, quel que soit le pays où le fait générateur du dommage se produit et quels que soient le ou les pays dans lesquels des conséquences indirectes de ce fait surviennent. ». Or l'identification de la loi du pays où le dommage survient peut s'avérer délicate. À titre d'exemple, la notation peut être émise par une agence située aux États-Unis, mais dont le travail d'analyse est effectué par une filiale européenne, alors que le titre faisant l'objet d'une notation est fondé sur des prêts immobiliers portant sur des immeubles situés en France et émis par une société d'un autre État selon

32. Sans préjudice de l'application du droit communautaire, tout recours visant des agences de notation de crédit en relation avec une violation des dispositions du présent règlement devrait être effectué conformément au droit national applicable en matière de responsabilité civile.

33. Les agences de notation de crédit mentionnées à l'art. L. 544-4 engagent leur responsabilité délictuelle et quasi délictuelle, tant à l'égard de leurs clients que des tiers, des conséquences dommageables des fautes et manquements par elles commis dans la mise en œuvre des obligations définies dans le règl. (CE) $n^{\circ}$ 1060/2009 du PE et Cons. du 16 septembre 2009, précité. Tout accord ayant pour effet de soumettre, par avance et exclusivement, aux juridictions d'un État tiers à l'UE un différend relatif aux dispositions du règl. (CE) $\mathrm{n}^{0}$ 1060/2009 du PE et Cons. du 16 septembre 2009, précité, alors que les juridictions françaises auraient été compétentes pour en connaître à défaut d'un tel accord, est réputé nul et non écrit.

34. Sabine Corneloup, «La place du règlement Rome II dans la régulation globale des marchés financiers ", Cahier de droit de l'entreprise, $\mathrm{n}^{\circ}$ 6, novembre 2012, doss. 30. 
une tierce $1 \mathrm{i}^{35}$. Cet exemple démontre à lui seul la difficulté qu'il peut y avoir à identifier la loi du lieu du dommage en cas de notation fautive. Sur cette question, la doctrine est divisée ${ }^{36}$.

Le règlement Rome II indique seulement qu'il convient de déterminer la loi applicable en fonction du lieu où le dommage survient et non du lieu où le délit a été commis (considérants 15 à 17). La loi du lieu où la notation a été effectuée doit donc être écartée. Cette loi serait de plus difficile à déterminer dès lors que «d'une manière générale, les équipes analytiques des agences sont constituées sur une base européenne, voire globale, et non uniquement nationale ${ }^{37}$ ".

Face à ces difficultés, une partie de la doctrine suggère l'élaboration d'une règle spécifique en matière de délits financiers. Le Conseil allemand pour le droit international privé a pu proposer une disposition englobant tous les délits financiers qui prévoit plusieurs rattachements principaux, corrigés ou suppléés par des rattachements subsidiaires ${ }^{38}$. La proposition envisage directement la question de la détermination de la loi applicable en cas de notation fautive d'un émetteur. Il est ainsi prévu un recours systématique à la clause d'exception. Une telle clause, prévue au sein du règlement Rome II (art. 4.3), permet d'écarter l'application de l'article 4.1 au profit de la loi qui présenterait des liens manifestement plus étroits au regard de l'ensemble des circonstances. Il est vrai que dans certaines situations, notamment comme celle décrite ci-dessus, le recours à la clause d'exception pourrait s'avérer pertinent. Si une partie de la doctrine se prononce en faveur de cette solution ${ }^{39}$, préconiser son recours quasi systématique, constituerait un détournement de la clause d'exception qui a vocation, comme son nom l'indique, à jouer seulement de manière ponctuelle. De plus, recourir à l'article 4.3 afin de déterminer la loi applicable à l'action en responsabilité à l'égard des agences de notation remettrait en cause de manière trop importante la prévisibilité

35. Exemple repris à Mathias Audit, op. cit., lui-même emprunté à Frédérick Lacroix, "L'échec des dispensateurs de confiance», Revue de droit bancaire, janvier 2009, étude 5 , spéc. $\mathrm{n}^{\mathrm{O}} 26$.

36. Matthias Lehmann, «Proposition d'une règle spéciale dans le règlement Rome II sur les délits financiers ", Revue critique de droit international privé, 2012, p. 485. Florian Denis, "La nouvelle responsabilité civile des agences de notation», Revue Lamy droit des affaires, $\mathrm{n}^{\circ} 65$, novembre 2011, p. 73.

37. Rapport 2009 de l'AMF sur les agences de notation, p. 42.

38. Version française disponible dans Revue critique de droit international privé, 2012, p. 679 et suiv.

39. Wolf Georg Ringe, Alexander Hellgardt, «The International Dimension of Issuer Liability - Liability and Choice of Law from a Transatlantic Perspective», Oxford Journal of Legal Studies, vol. 31, 2011, p. 23. 
et la sécurité juridique des parties, ce qui irait à l'encontre des objectifs fixés par le législateur européen. Dès lors, d'autres solutions doivent être recherchées.

\subsubsection{La loi du centre des intérêts de la victime comme loi du lieu du dommage}

Les dommages résultant d'une notation fautive sont essentiellement des dommages économiques ou patrimoniaux, dès lors il serait tentant d'identifier le lieu du dommage comme étant celui du centre d'administration du patrimoine de la victime, c'est-à-dire du siège de l'émetteur ou du domicile de l'investisseur ${ }^{40}$. Or, dans l'hypothèse où plusieurs investisseurs seraient victimes d'une notation fautive, chaque demande serait soumise à une loi différente bien que le dommage ait été causé par la même notation. Toutefois, la possibilité d'introduire une action collective pourrait constituer une solution satisfaisante afin de régler cette difficulté ${ }^{11}$. De plus, la possible dispersion du litige entre plusieurs États membres n'a pas empêché la Cour de justice de l'Union européenne (CJUE) de retenir une solution ayant des conséquences analogues en matière de détermination de la compétence juridictionnelle. En effet, dans une affaire Kronhofer ${ }^{42}$, la CJUE a interprété l'article 5.3 du règlement 44/2001 relatif à la détermination de la compétence juridictionnelle en cas de dommage comme étant celui du lieu où la partie du patrimoine a été endommagée, ce qui peut conduire à un éclatement du litige. Cette solution devrait prévaloir en ce qui concerne la détermination de la juridiction compétente pour connaître de l'action en réparation intentée à l'encontre d'une agence de notation, le règlement 462/2013 précisant que «la juridiction compétente pour connaître d'une action en responsabilité civile intentée par un investisseur ou un émetteur est déterminée selon les règles applicables du droit international privé » (art. 35 bis 4 in fine). Retenir une interprétation identique en matière de détermination de la loi applicable aurait l'avantage de permettre que le juge saisi applique sa propre loi. On pourra cependant objecter que les solutions adoptées en matière de détermination de la compétence juridictionnelle n'ont pas nécessairement vocation à être transposées en

40. Matthias Lehmann, op. cit., p. 485 , spéc. $\$ 11$ et suiv.

41. Sabine Corneloup, art. cité.

42. CJUE, 10 juin 2004, aff. C-168/02, Revue critique de droit international privé 2005, p. 326, note Horatia Muir Watt. 
matière de conflits de lois ${ }^{43}$. Cependant, retenir la compétence de la loi du domicile de l'investisseur ou du siège de l'émetteur nous paraît être en adéquation avec la double fonction ${ }^{44}$ poursuivie par les règles de responsabilité civile des agences de notation. D’une part, le législateur européen entend clairement favoriser un droit au recours des victimes de notations fautives, sans pour autant léser de manière trop importante les agences (considérants $32^{45}$ et $35^{46}$ ). La responsabilité civile des agences de notation est alors perçue comme un outil de protection des intérêts privés. Interpréter la notion de «lieu du dommage» comme renvoyant au siège ou au domicile des victimes assure l'application de leur droit national, ce qui constitue une faveur et assure la prévisibilité pour ces dernières. Le droit ainsi désigné ne sera pas nécessairement le plus enclin à favoriser la réparation des investisseurs et des émetteurs victimes, ce qui permet de préserver un certain équilibre avec les intérêts des agences. D'autre part, les règles relatives à la responsabilité civile des agences de notation doivent également être appréhendées comme un outil de régulation de leur activité. La responsabilité civile des agences complète alors les sanctions administratives mises en place par le règlement 1060/2009. Retenir la loi du domicile ou du siège de la victime de la notation fautive favorise les demandes d'indemnisation, ce qui contribue à assurer une meilleure mise en œuvre de la réglementation puisque les agences de notation seront enclines à davantage de diligence afin d'éviter d'éventuelles condamnations.

Cette interprétation des règles de conflits européennes n'occulte pas le constat selon lequel le régime de responsabilité civile des agences de notation mit en place par le droit européen demeure lacunaire et manque d'efficacité. L'Union européenne est certes parvenue à imposer un cadre réglementaire en mettant en place un système d'enregistrement et de surveillance des agences qui souhaitaient exerçaient leur activité sur le territoire européen. Toutefois, édicter un régime de responsabilité

43. Tristan Azzi, «Bruxelles I, Rome I, Rome II : regard sur la qualification en droit international privé communautaire », Dalloz, 2009, p. 1621.

44. Infra, $\mathrm{n}^{\mathrm{o}} 2$.

45. "Les investisseurs et les émetteurs ne sont pas toujours en mesure d'invoquer la responsabilité des agences de notation de crédit à leur égard. Il peut s'avérer particulièrement difficile d'établir la responsabilité civile d'une agence de notation de crédit en l'absence de lien contractuel entre celle-ci et, par exemple, un investisseur ou un émetteur noté de manière non sollicitée.»

46. «En particulier, les États membres devraient avoir la possibilité de maintenir les régimes nationaux en matière de responsabilité civile qui sont plus favorables aux investisseurs ou aux émetteurs, ou qui ne sont pas fondés sur une infraction au règlement $(\mathrm{CE}) \mathrm{n}^{\mathrm{o}}$ 1060/2009." 
européen renvoyant aux droits nationaux sur un nombre important de questions favorise le law shopping. Le règlement 432/2013 ne fait ainsi que masquer les différences persistantes entre les législations nationales sur la question de la responsabilité civile des agences de notation ${ }^{47}$. Le Brexit n'a fait qu'aggraver ce constat. Le droit anglais étant déjà prisé par les agences de notation ${ }^{48}$, il fait peu de doute que les autorités britanniques réforment leur droit national en décidant d'opter pour un très faible encadrement des agences afin de favoriser l'attractivité de leur marché ${ }^{49}$. Face à la concurrence des législations nationales et à l'emprise mondiale des agences, il est essentiel d'adopter des mesures au niveau européen et international capables d'assurer une responsabilité effective de celles-ci en cas de notation fautive.

\section{Pour l'instauration d'une obligation de diligence des agences de notation au niveau international}

Différents obstacles rendent difficile la mise en place d'un véritable régime harmonisé de responsabilité civile des agences de notation (2.1). Face à ces difficultés, l'établissement d'une obligation de diligence des agences pourrait s'avérer un moyen efficace d'assurer un dédommagement des victimes de notations fautives (2.2).

\subsection{Les difficultés liées à l'établissement de la responsabilité civile des agences de notation}

La responsabilité civile des agences de notation serait difficile, voire introuvable ${ }^{50}$, plusieurs obstacles s'opposant à sa mise en œuvre. Les agences tentent d'échapper à la mise en cause de leur responsabilité en se fondant principalement sur deux moyens. D'une part, en ayant recours à des clauses limitatives ou exclusives de responsabilité (2.1.1). D'autre part, en affirmant que les notations ne seraient que de simples opinions (2.1.2).

47. Matthias Lehmann, «Civil liability of rating agencies - an insipid sprout from Brussels », Capital Markets Law Journal, vol. 11, nº 1, 2016, p. 60.

48. Samuel Kern Alexander, «Tort Liability for Ratings of Structured Securities under English Law», University of Oslo Faculty of Law Legal Studies Research Paper, $n^{\circ}$ 2015-06.

49. Daniel Cash, "Credit Rating Agency Regulation after the UK's European Union Membership Referendum », 37 The Company Lawyer 10, 2016.

50. Frédérique Espagnac et Aymeri de Montesquiou, Agence de notation: pour une profession réglementée, Rapport du Sénat du 18 juin 2012, nº 598, p. 154. 


\subsubsection{Le recours aux clauses limitatives et exclusives de responsabilité}

Les agences de notation ont l'habitude, par le jeu de clauses, de limiter ou d'exclure leur responsabilité en cas de pertes dues à une notation ${ }^{51}$. Ces clauses peuvent figurer dans le contrat conclu entre l'émetteur et l'agence, mais également dans les conditions générales d'utilisation des notations figurant sur les sites internet des agences ${ }^{52}$. La portée de ces clauses dépasse le cadre des contrats conclus entre les agences et les émetteurs. En effet, l'utilisation des notations figurant sur les sites des agences nécessite une inscription et la validation des conditions générales d'utilisation, si bien qu'un investisseur qui fonderait sa décision d'investir en s'appuyant sur une notation référencée sur le site d'une agence se verrait imposer ses conditions générales d'utilisation. La question de l'efficacité de ces clauses se pose également en ce qui concerne l'investisseur qui aurait connaissance d'une notation lorsqu'elle est publiée dans un journal. Dans cette dernière hypothèse, l'investisseur n'aurait jamais eu l'occasion de prendre connaissance de l'existence des conditions générales du site, si bien qu'il est peu probable

51. Eddy Wymeersch et Marc Kruithof, "Regulation and liability of credit rating agencies under Belgian Law», The Belgian reports to the 2006 Utrecht Congress of the International Academy of Comparative Law, Bruylant, mars 2016, spec. p. 18

52. Les conditions générales d'utilisation du site internet de Moody's précise notamment: "Dans la mesure où cela est autorisé par la loi, les parties Moody's ne sauraient encourir une quelconque responsabilité du fait de tout préjudice subi qu'il soit exceptionnel, indirect, incident ou par ricochet résultant de ou en relation avec votre accès ou votre utilisation de ce site et de son contenu, et ce, même dans l'hypothèse où l'une des parties Moody's aurait été avisée de l'éventualité de la survenance d'un préjudice, ce qui inclut, sans être limité à : (I) une indemnisation, un remboursement ou des dommages intérêts résultant de la perte de profits actuels ou en prospective (...) (VII) les réclamations de tout tiers (VIII) tout autre préjudice survenant en lien ou sans lien avec toute autre circonstance. / Dans les limites autorisées par la loi les parties Moody's déclinent toute responsabilité pour le dommage direct ou compensatoire causé à toute personne ou entité résultant notamment de toute négligence (à l'exclusion de la fraude, de la faute lourde ou toute autre type de responsabilité qui pour lever toute ambigüité ne peuvent être exclues légalement ou toute contingence dans ou hors contrôle des parties Moody's qui surgirait lors de ou en relation avec votre utilisation du site et de ses contenus en tout état de cause dans les limites autorisées par la loi, le montant maximal, dont les parties Moody's pourraient être redevables pour quelque motif que ce soit du fait de l'accès à ou l'utilisation du site ou de ses contenus ne saurait excéder la plus élevées des sommes suivantes: soit (A) le montant total payé par l'utilisateur pour les services fournis par ce site au titre des présentes modalités d'utilisation durant les 12 mois précédant la survenance de l'événement donnant lieu à la mise en jeu de la responsabilité soit (B) 100 U.S. \$.» 
qu'un juge décide de les lui imposer. L'efficacité des clauses limitatives de responsabilité ne va pas nécessairement à l'encontre de la mise en place d'une responsabilité effective des agences. Il est essentiel que cette dernière ne conduise pas à mettre en danger l'activité de la notation en confrontant les agences à une "responsabilité potentiellement illimitée » (considérant 33 in fine). Permettre l'efficacité des clauses limitatives de responsabilité, sous certaines conditions, pourrait parvenir à éviter un tel écueil. Il s'avère que l'efficacité de ces clauses va dépendre du droit national applicable.

Jusqu'à l'adoption du règlement 462/2013, la question pouvait être abordée de manière très différente entre les législations nationales, ce qui favorisait le law shopping. À titre d'exemple, l'article L. 544-6 du Code monétaire et financier français répute non écrites les clauses par lesquelles les agences excluent leur responsabilité ${ }^{53}$. Après l'adoption de cette disposition, Moody's a demandé systématiquement à tous les émetteurs français de signer des contrats soumis au droit britannique afin de contourner l'interdiction française ${ }^{54}$. Désormais, l'article $35 \mathrm{bis}$ $\$ 3$ du règlement $462 / 2013$ dispose :

La responsabilité civile de l'agence de notation de crédit visée au paragraphe 1 ne peut être limitée a priori que lorsque cette limitation est: raisonnable et proportionnée et autorisée par le droit national applicable conformément au paragraphe 4. Toute limitation qui ne satisferait pas au premier alinéa, ou toute exclusion de responsabilité civile n’a aucun effet juridique.

Les clauses exclusives de responsabilité n'ont donc plus aucun effet juridique sur le territoire européen. Toutefois, le règlement laisse une large place au pouvoir d'interprétation du juge en ce qui concerne les clauses limitatives de responsabilité. Or, il est à craindre que le juge saisi considère souvent ces dernières comme «raisonnables et proportionnées" dès lors que le règlement lui-même reconnait la difficulté inhérente à l'exercice de notation (considérant 35). Le juge saisi risque d'être fortement influencé par son propre droit national, ce qui au regard des différences existantes entre les différents régimes ${ }^{55}$ fait naître une forte insécurité juridique.

53. «Les clauses qui visent à exclure la responsabilité des agences de notation de crédit mentionnées à l'article L. 544-4 sont interdites et réputées non écrites. »

54. Rapport d'information du Sénat français $\mathrm{n}^{\circ} 598$, "Agence de notation: pour une profession réglementée », 11 juin 2012.

55. Philippe Pierre et Fabrice Leduc (dir.), La réparation intégrale en Europe. Études comparatives des droits nationaux, Bruxelles, Larcier, 2012. 
Les moyens utilisés par les agences pour s'opposer à la mise en œuvre de leur responsabilité civile ne s'arrêtent pas seulement aux clauses limitatives de responsabilité. Elles ont également recours à l'argument récurrent selon lequel les notations ne constitueraient que de simples opinions.

\subsubsection{Les notations représentent plus que de simples opinions}

Pour parvenir à un droit de l'Union plus efficace en matière de responsabilité des agences de notation, il est essentiel de dépasser une autre difficulté liée à la nature même de l'activité de notation. Afin d'éviter que leur responsabilité ne soit engagée, les agences ont pour habitude, que ce soit dans leurs contrats ou dans les conditions générales d'utilisation des notations, de préciser que les notations constituent de simples opinions ${ }^{56}$. Il s'agit d'un obstacle important à l'établissement de la responsabilité des agences, notamment aux ÉtatsUnis. En affirmant que les notations ne sont que simples opinions, ces dernières devraient relever de la protection du premier amendement de la Constitution américaine ${ }^{57}$, lequel vise à assurer une large liberté de parole et d'opinion. Les notations devraient dès lors être protégées par le droit à la liberté d'expression au même titre que la liberté de la presse ${ }^{58}$, la doctrine allant même jusqu'à comparer les notations aux «plus courts

56. À titre d'exemple, Moody’s précise: «Vous consentez expressément que les notations de crédit et autres opinions fournies via le Site sont, et doivent uniquement être interprétées comme l'expression d'une opinion sur les risques de crédit futurs relatifs d'entités, d'engagements de crédits et de titres de dettes et non pas comme une affirmation présente ou passée de solvabilité, de conseils financiers ou d'investissement, des recommandations pour des décisions d'accorder un crédit ou des décisions d'achat, de détention ou de vente de valeurs mobilières, un engagement quant à l'exactitude des données ou conclusions et ni comme une tentative d'évaluer de manière indépendante ou de se porter garant de la situation financière d'une société.»

57. «Le Congrès n'adoptera aucune loi relative à l'établissement d'une religion, ou à l'interdiction de son libre exercice; ou pour limiter la liberté d'expression, de la presse ou le droit des citoyens de se réunir pacifiquement ou d'adresser au Gouvernement des pétitions pour obtenir réparations des torts subis. »

58. Régis Bismuth, "Agences de notation, dette souveraine et liberté d'expression - Quelques réflexions sur la légalité (et l'opportunité) d'un contrôle européen des notations souveraines non sollicitées", dans Geneviève Dufour et David Pavot (dir.), La crise des dettes souveraines et le droit: Approches croisées Canada-Europe, Montréal, LexisNexis, 2014, p. 87. Frédéric Schneider, «Les agences de notation et les pouvoirs publics », Revue du droit public, $\mathrm{n}^{\circ}$ 1, 2015, p. 83 . 
éditoriaux du monde ${ }^{59} »$. Si l'on retient que les notations ne sont que de simples opinions protégées par la liberté d'expression, toute action en responsabilité civile visant une agence de notation ne pourrait aboutir, dès lors qu'aucune intention malveillante de la part de cette dernière à l'encontre du plaignant ne serait établie. S'il est vrai qu'il peut exister une certaine analogie entre l'activité des agences de notation et le journalisme (un travail de recherche d'informations, d'évaluation et de synthèse), et même si la jurisprudence américaine s'est longtemps prononcée en ce sens ${ }^{60}$, il n'apparaît pas pertinent d'assimiler les notations à de simples opinions. Dans une décision du 2 septembre 2009, puis dans une décision du 24 mai 2010, il a été affirmé qu'une agence de notation ne pouvait se prévaloir $\mathrm{du}_{1}{ }^{\mathrm{er}}$ amendement dans le cadre de l'émission de notes sur des produits financiers ${ }^{61}$. Plus récemment, à la suite d'une plainte du ministère de la Justice américaine, Standard and Poor's a accepté de payer une amende de 1,37 milliard de dollars afin d'éviter un procès. Ces quelques décisions n'épuisent pas le débat, cependant, elles marquent assurément un tournant en matière de responsabilité civile des agences de notation.

En outre, même en considérant que les notations sont assimilables à de simples opinions, cela ne devrait pas conduire à écarter systématiquement la responsabilité civile des agences. Ces dernières bénéficient d'un pouvoir certain, tenant aussi bien au rôle central que leurs notations continuent à jouer dans l'économie mondiale ${ }^{62}$, qu'à la concentration de l'industrie de la notation. En effet, les trois agences américaines Standard \& Poor's, Fitch et Moody's détiennent plus de $90 \%$ du marché de la

59. Gregory Husisian, «What Standar of Care Should Govern the World's Shortest Editorials? An Analysis of Bond Rating Agency Liability», Cornell Law Review, vol. 75, 1990, p. 411. David Grais, Kostas Katsiris, «Not the "World's Shortest Editorial" : Why the First Amendment does not shield the Rating Agencies from liability for over-rating CDOs », Bloomberg Law Reports, novembre 2007.

60. Hubert de Vauplanne, op. cit., p. 450 et 451.

61. Abu Dhabi Commercial Bank c. Moody's and Morgan Stanley, Southern District Court of New York, 2 septembre 2009. California Public Employees' Retirement Systems v. Moody's Corp. et al., Superior Court of California, San Francisco County, $\mathrm{n}^{\circ}$ 09-490241, 24 mai 2010.

62. L'Union se fixe comme objectif de supprimer l'ensemble des références aux notations de crédit à des fins réglementaires, sous réserve d'avoir identifié et mis en œuvre des solutions alternatives à l'évaluation du risque de crédit appropriées, considérant 6 du règlement $n^{\circ} 462 / 2013$. 
notation et sont les seules à opérer au niveau mondial ${ }^{63}$. Compte tenu de l'important recours aux notations dans la réglementation mondiale des marchés et de l'existence d'un oligopole des agences, les notations se voient attribuer une valeur qui dépasse largement la simple opinion. Même dans l'hypothèse où un investisseur douterait de la qualité d'une notation, celui-ci n'aurait pas d'incitation à suivre son propre jugement, compte tenu de l'impact des notations sur le marché et du comportement mimétique des autres investisseurs à leur égard ${ }^{64}$.

Les notations influencent de manière significative les décisions d'investissement, ainsi que l'image et l'attrait d'un émetteur sur le plan financier. Les agences de notation le reconnaissent elles-mêmes: valeur de référence pour le placement, large accès aux capitaux, meilleure flexibilité financière et entretien de la confiance des investisseurs, sont autant d'arguments mis en avant par les agences afin de faire la promotion des notations ${ }^{65}$. Elles sont fondées sur des données financières et informations auxquelles les investisseurs n'ont pas accès, si bien qu'elles contribuent considérablement à réduire l'asymétrie de l'information entre acteurs du marché ${ }^{66}$. Au même titre qu'un certain nombre d'autres professions, les agences de notation doivent être tenues responsables des informations trompeuses qu'elles pourraient véhiculer. Qu'il s'agisse du commissaire aux comptes qui participe à l'information du public ${ }^{67}$, du journaliste économique ${ }^{68}$ ou des analystes financiers ${ }^{69}$, tous ont pu voir leur responsabilité engagée pour avoir diffusé des informations inexactes ou trompeuses. Or, la notation, même en étant assimilée à

63. US House of Representatives, Approaches to Improving Credit Rating Agency Regulation, Hearing Before the Subcommittee on Capital Markets, Insurance and Government Sponsored Enterprises of the Committee on Financial Services, $\mathrm{n}^{\circ} 111-33$, 19 mai 2009, p. 16.

64. Jean-Marc Gollier, «Le courage de la vérité dans un monde mimétique», dans Brunot Colmant (dir.), op. cit., p. 143. Régis Bismuth, op. cit., p. 109.

65. Voir par exemple le site internet de Moody's: https://www.moodys.com/pages/ default_fr.aspx.

66. En ce sens, Commission Staff Working Document accompanying document to the Proposal for a Regulation of the European Parliament and the Council on Credit Rating Agencies, 12 novembre 2008, COM (2008) 704.

67. Cass. com., 11 juil. 2006 (deux arrêts) : Bull. Joly Bourse 2006, p. 443, $\$ 102$, note Bernard Garrigue et Sébastien Bonfils. Bull. Joly Sociétés 2006, p. 1357, \$284, note Thierry Granier. Hubert de Vauplane, Banque et droit, 2006, $\mathrm{n}^{\circ}$ 109, p. 60. Yann Paclot, Journal des sociétés, 2006, $\mathrm{n}^{\circ} 37, \mathrm{p} .66$.

68. CA Paris, 14 déc. 2005 : RTD fin., 2006, p. 136, obs. Nicolas Rontchevsky.

69. CA Paris, 30 juin 2006: D., 2006, p. 2241, obs. Xavier Delpech; Banque et droit, 2006, $\mathrm{n}^{\mathrm{o}}$ 4, obs. Hubert de Vauplane; RTD com., 2007, p. 875. Nicolas Rontchevsky et Michel Storck; Bull. Joly Bourse 2006, p. 594, \$141, note Dominique Schmidt. 
une opinion, revêt une valeur informationnelle puisqu'elle est fondée sur des données financières, afin d'estimer la probabilité de défaut d'un produit. La Cour d'appel de Paris, dans un arrêt très remarqué, a pu retenir à l'égard des analystes financiers que «la faute, quasi délictuelle, emporte la violation des devoirs de prudence ou d'objectivité, corollaire de la liberté d'exprimer une opinion, sans qu'il soit nécessaire d'établir une intention de nuire ${ }^{70} »$. Les agences de notations devraient ainsi plus largement être soumises à des principes d'objectivité, d'impartialité, de rigueur et de prudence, qui en cas de manquement caractérisé devraient conduire à retenir leur responsabilité. Soumettre les agences de notation à une obligation de diligence permettrait ainsi, en cas de manquement, d'assurer une réparation effective en cas de notation trompeuse.

\subsection{La mise en place d'une obligation de diligence des agences de notation}

Intégrer une obligation de diligence des agences de notation au sein du règlement 462/2013 renforcerait l'efficacité du régime de responsabilité mis en place par le législateur européen. Elle étendrait les fondements des actions en réparation au-delà des seuls manquements aux dispositions du règlement 1060/2009 (2.2.1). Elle permettrait également de contourner les principales difficultés liées à l'établissement de la responsabilité civile des agences, tout en assurant un équilibre certain entre les différents intérêts en présence (2.2.2).

\subsubsection{Une obligation de diligence assurant une entière responsabilité des agences}

Soumettre les agences de notation à une obligation de diligence permettrait d'étendre les fondements sur lesquels la responsabilité civile de ces dernières pourrait être recherchée. Au-delà de la violation des obligations du règlement 1060/2009, la responsabilité civile délictuelle des agences pourrait être recherchée sur le fondement d'un manque de prudence et de diligence normalement attendus. L'intérêt est certain. Comme en droit français, les manquements éventuels des agences ne seraient pas seulement appréciés à l'aune de la réglementation européenne, mais également au regard du code de bonne conduite élaboré par l'Organisation internationale des commissions de valeurs (OICV). Publié

70. Ibid. 
en 2004, ce code, considéré comme l'outil de référence en matière de régulation des agences de notation, énonce des mesures regroupées autour de quatre thèmes : la qualité et l'intégrité de la notation, la prévention des conflits d'intérêts, la responsabilité des agences envers les investisseurs et les émetteurs, ainsi que le traitement d'informations confidentielles par les agences. En 2006, un accord a été trouvé entre le régulateur européen et les agences de notation afin d'appliquer sur une base volontaire le code OICV. Ce dernier a été révisé en 2008 et 2015, en introduisant différentes modifications destinées à renforcer les dispositions concernant la protection de l'intégrité du processus de notation, la gestion des conflits d'intérêts et en ajoutant des dispositions concernant la gouvernance, la formation et la gestion du risque. Si les dispositions de ce code ont largement inspiré la réglementation européenne, certaines d'entre elles, parfois fondamentales, en sont absentes. Tel est le cas notamment en ce qui concerne la nécessité d'avoir un nombre suffisant d'analystes au sein des agences. Or, il s'agit précisément de l'une des causes de l'échec des agences de notation. Retenir une obligation de diligence des agences permettrait de caractériser une faute de ces dernières, dans l'hypothèse où elles ne disposeraient pas de ressources suffisantes pour assurer leur activité $^{71}$.

Le législateur australien s'est également prononcé en faveur d'une obligation de diligence des agences de notation, et a entièrement harmonisé l'encadrement de ces dernières autour du code $\mathrm{OICV}^{72}$. Une agence dérogeant à ce dernier est donc dorénavant tenue d'expliquer et justifier les raisons de sa non-conformité aux dispositions du code et pourra éventuellement être condamnée à réparation. L'affaire Baturst offre un exemple d'application de ces dispositions ${ }^{73}$.

Dans cette affaire, Standard and Poor's $(S \& P)$ a été condamnée à dédommager à hauteur de 30 millions de dollars australiens douze municipalités. Ces dernières avaient perdu des millions de dollars après

71. En ce sens, Anastasia Sotiropoulou, «La responsabilité civile des agences de notation ", Bull. Joly Bourse 2013, n ${ }^{\circ}$ 1, p. 47, spéc. $\$ 17$.

72. "Credit rating agencies - guidance on certain AFS licence conditions", http://asic.gov.au/regulatory-resources/financial-services/credit-rating-agencies/ credit-rating-agencies-guidance-on-certain-afs-licence-conditions/.

73. Federal Court of Australia, Bathurst Regional Council v. Local Government Financial Services Pty Ltd $\left(n^{0} 5\right)$ [2012] FCA 1200, 5 novembre 2012. Pour une présentation sommaire de cette décision : Rommel Harding-Farrenberg et Kieran Donovan, "Case Note : Duty of Care, Rating Agencies and the "Grotesquely Complicated" Rembrandt : Bathurst Regional Council v. Local Government Financial Services Pty Ltd $\left(\mathrm{n}^{\circ}\right.$ 5) », Business Law International, vol. 14, $\mathrm{n}^{\circ}$ 2, mai 2013, p. 185-194. 
avoir investi dans des titres financiers notés «AAA » qui avaient perdu $90 \%$ de leur valeur entre 2006 et 2008. Les juges australiens ont considéré que la notation attribuée à ces produits était trompeuse et qu'elle était basée sur des informations inexactes. $S \& P$ aurait fait preuve de négligence en ne prenant pas la peine de vérifier les informations fournies par la banque qui a émis les titres alors que ces produits étaient nouveaux. Un courriel d'un employé de $S \hookleftarrow P$ révélait que les avis des analystes sur la qualité du produit noté avaient pu être très divergents, mais que malgré les doutes, $S \hookleftarrow P$ n'avait pas décidé de modifier sa notation. En appel, $S \& P$ a admis que le triple $A$ était une notation viciée, mais a fait valoir qu'elle n'avait aucune obligation de diligence dès lors que les conditions d'une telle obligation n'étaient pas réunies. L'agence a notamment fait valoir que les investisseurs ne pouvaient pas être perçus comme des personnes vulnérables et auraient dû se renseigner davantage avant de prendre leur décision d'investir. La Cour rejette cette affirmation en considérant que la vulnérabilité des intimés résulte de la complexité du produit financier en cause. Dès lors, ces derniers n'étaient pas en leur capacité d'analyser les produits en cause et dépendaient exclusivement de la notation de $S \hookleftarrow P$. Autrement dit, la responsabilité d'une agence sur le fondement d'un manquement à son obligation de diligence ne devrait pouvoir être retenue qu'en ce qui concerne les produits financiers complexes ou lorsque la notation constitue l'unique source d'information à l'égard de ces derniers.

Pour certains ${ }^{74}$, le risque de voir une situation analogue à celle de l'affaire Bathurst se produire désormais en Europe serait inexistant, eu égard aux différentes obligations qui incombent aux agences de notation, notamment en ce qui concerne la qualité des méthodes de notation et la diffusion d'information ${ }^{75}$. Toutefois, l'AEMF reconnait elle-même que sa surveillance à l'égard des méthodes de notation est insuffisante, mais également que les exigences en la matière édictées par le règlement 1060/2009 ne sont pas toujours claires ${ }^{76}$. Dès lors, le

74. Edith Weemaels, «La réglementation des agences de notation en Europe: quand l'inutile affaiblit le nécessaire», Rev. Prat. Soc., $\mathrm{n}^{\mathrm{o}} 3$, 2013, Bruylant, p. 279, spéc. p. 335 et 336.

75. Règlement délégué 447/2012 de la Commission du 21 mars 2012 complétant le Comm. CE, règl. (CE) n ${ }^{\circ}$ 1060/2009 du du PE et Cons. par des normes techniques de réglementation aux fins de l'évaluation de la conformité des méthodes de notation de crédit, JOUE $n^{\circ}$ L 140, 30 mai 2012, p. 14.

76. Rapport spécial $n^{0} 22$ de la Cour des comptes européenne: «La surveillance par l'UE, des agences de notation de crédit est bien en place, mais elle n'est pas encore totalement efficace», 2015, spéc. p. 32 et suiv. 
risque de voir réapparaitre des notations fautives persiste. De plus, la surveillance exercée par l'AEMF concernant les méthodes de notation ne couvre pas tous leurs aspects ${ }^{77}$. Dès lors, l'établissement d'une obligation de diligence à l'égard des agences de notation est essentiel.

\subsubsection{Du juste équilibre entre préservation de l'activité des agences et nécessaire réparation en cas de notation fautive}

Une responsabilité civile élargie des agences ne risque-t-elle pas de remettre en cause l'activité de la notation? Pour une partie de la doctrine ${ }^{78}$, prévoir une responsabilité des agences serait incompatible avec l'activité de la notation. Il est vrai que cette dernière revêt un aspect prédictif certain, si bien qu'il est parfois difficile d'établir une distinction entre une faute de l'agence et une simple notation incorrecte. Par exemple, il n'est pas pertinent de retenir une faute de l'agence si la dégradation de la situation d'un émetteur est due à des changements de circonstances économiques imprévisibles. La notation fautive devrait ainsi s'entendre comme le manquement d'une agence de notation à son obligation de diligence ayant conduit à produire une déclaration inexacte entraînant des pertes purement économiques. Cette approche nous paraît en conformité avec la nature même de l'activité de la notation : l'agence n'a pas à assurer une notation certaine pour l'avenir, mais simplement à faire preuve d'une diligence raisonnable lors de son travail d'analyse et d'émission. La preuve d'une faute de l'agence devrait toujours être à la charge du plaignant. Toutefois, une perte de valeur soudaine ou une dégradation brutale d'un "AAA " peuvent constituer de sérieux indices.

L'établissement d'un lien de causalité entre le dommage subi par l'investisseur ou l'émetteur et le manquement à l'obligation de diligence de l'agence devrait parfois être assez simple à établir. Tel sera notamment le cas en ce qui concerne les produits financiers complexes dont la notation constitue l'unique source d'information et pour lesquels un investisseur seul ne peut avoir une vision véritablement éclairée ${ }^{79}$. Un manquement à l'obligation de diligence pourrait également servir de fondement à l'action des investisseurs institutionnels auxquels la

77. Ibid.

78. Norbert Gaillard, "Remettre la notation financière à sa juste place», Institut Montaigne, juillet 2012 ; Edith Weemaels, op. cit., p. 279 ; JCP G, $\mathrm{n}^{\circ} 37,12$ septembre 2013, note 1502, Morgan Marechal et Marie Razafindrakoto, «Les conséquences de la qualification des notations financières sur la responsabilité des agences ».

79. Supra. 
législation impose de détenir dans leur portefeuille des produits ayant une notation élevée ${ }^{80}$ et qui n'ont ainsi pas d'autres choix que de se référer aux notations.

Si les notations constituent des informations importantes pour les investisseurs et plus généralement pour l'ensemble des acteurs des marchés financiers, ces derniers devraient davantage être enclins à fonder leurs décisions sur leurs propres opinions ${ }^{81}$. Nombre d'investisseurs se fient aux agences de notation, parce qu'ils ne disposent pas de l'expertise ou des ressources nécessaires (en matière de temps et d'argent) pour procéder à leur propre analyse du risque de crédit $^{82}$. Toutefois, des investissements réguliers et diversifiés peuvent engendrer des bénéfices plus importants que l'acquisition de titres sur la base d'information ${ }^{83}$. Ainsi, si les agences de notation devaient être soumises à une obligation de diligence, investisseurs et émetteurs devraient être tenus à une obligation de vigilance. Cette dernière devrait relever de l'appréciation souveraine des juges étant donné que de petits investisseurs ne sont pas toujours en mesure de réexaminer de façon critique les notations, ce que le règlement 462/2013 reconnait lui-même (considérant 36 in fine).

La mise en place d'un régime spécial de responsabilité fait craindre une multiplication des condamnations, qui à terme pourrait conduire à une faillite des agences ${ }^{84}$. Si l'instauration d'un tel régime est essentielle pour assurer une réparation effective du préjudice causé aux victimes de notations fautives, encore faut-il que ces réparations soient encadrées, afin d'éviter que l'activité de notation ne soit mise en péril. Deux solutions semblent envisageables. Une première solution consisterait à prévoir une disposition qui limiterait la réparation des agences à un multiple de leurs honoraires annuels en cas de condamnation. Les agences pourraient également être tenues de souscrire une assurance afin de se prémunir contre d'éventuelles condamnations et répondre à leur besoin de réserve de capital ${ }^{85}$.

80. Supra.

81. Jean-Marc Gollier, «Le courage de la vérité dans un monde mimétique», dans Brunot Colmant (dir.), op. cit., p. 143, spéc. p. 157 et suiv.

82. Proposition du PE et Cons. sur les agences de notation de crédit, 12 novembre 2008, COM (2008) 704 final, p. 9.

83. Alexandre Delalgue, Fama, Hansen et Shiller, prix Nobel d'économie 2013, 14 octobre 2013, http://blog.francetvinfo.fr/classe-eco/2013/10/14/fama-hansenet-shiller-prix-nobel-deconomie-2013.html.

84. Floyd Norris, "In Actions, S\&P. Risked Andersen's Fate», The New York Times, 7 février 2013.

85. Jeffrey Manns, «Downgrading Rating Agency Reform», The George Washington Law Review, $\mathrm{n}^{\circ}$ 81, 2013, p. 101, spéc. p. 161. 
L'Union européenne n'est pas parvenue à mettre en place un régime de responsabilité des agences de notation efficace. Le régime de responsabilité demeure incomplet en ce qu'il sanctionne seulement les manquements au règlement 1060/2009 et repose sur le droit national en ce qui concerne un nombre important de questions, ce qui suscite une insécurité juridique importante. Imposer une obligation de diligence des agences de notation au niveau européen, en se fondant sur les dispositions du code OICV, permettrait de dépasser les difficultés liées à la détermination du droit national applicable, en assurant un alignement des solutions au plan international. Cette solution permettrait également de préserver un certain équilibre entre une sécurité nécessaire à l'activité des agences et un élargissement des fondements sur lesquels peuvent être demandés des dommages-intérêts, en réparation du préjudice causé par une notation fautive. Enfin, cela permettrait de mettre en place un régime de responsabilité utile en incitant les agences à l'autodiscipline, en renforçant l'efficacité de la réglementation, la confiance du public dans les agences et donc leur légitimité.

Docteur en droit Juriste assistant civil à la Cour d'appel de Rouen 